\title{
Integrated Cu-based TM-pass Polarizer using CMOS Technology Platform
}

\author{
Tien K. Ng, Zahed M. Khan, and Boon S. Ooi* \\ Division of Physical Sciences and Engineering \\ King Abdullah University of Science \& Technology (KAUST), Thuwal 23955-6900, Saudi Arabia. \\ *email: boon.ooi@kaust.edu.sa
}

\begin{abstract}
A transverse-magnetic-pass (TM-pass) copper $(\mathrm{Cu})$ polarizer is proposed and analyzed using the previously published two-dimensional Method-of-Lines beam-propagation model. The proposed polarizer exhibits a simulated high-pass filter characteristics, with $\mathrm{TM}_{0}$ and $\mathrm{TE}_{0}$ mode transmissivity of $>70 \%$ and $<5 \%$, respectively, in the wavelength regime of $1.2-1.6 \mu \mathrm{m}$. The polarization extinction ratio (PER) given by $10 \log _{10}\left(P T M_{0}\right) /\left(P T E_{0}\right)$ is $+11.5 \mathrm{~dB}$ across the high-pass wavelength regime. To the best of the authors' knowledge, we report here the smallest footprint CMOSplatform compatible TM-polarizer.
\end{abstract}

\section{INTRODUCTION}

Since the first proposal of photonic integrated circuits (PIC) [1], various passive and active components have been gradually developed with the eventual goal of monolithically integrate the components on the silicon or silicon-on-insulator (SOI) platform. The silica or silicon passive components include the $\mathrm{V}$-groove and taper waveguides, optical splitters, array waveguide gratings, ring resonators; while the non-siliconbased passive components include the III-V based waveguides, polymer waveguides, and whispering gallery [2]. The active components include semiconductor optical amplifiers (SOA), detectors and emitters. External carrier or electric field can also be applied to the passive components to change their bandgap and optical constants, leading to active device application, such as electroabsorption modulators. A recent advancement in the active optical components research involves the use of broadwavelength gain material structure, such as intermixed quantum-dots (QD) [3] and as-grown quantum-dash (QDash) [4] material structure where a broad electroluminescent (EL) linewidth were achieved for such emitters. The availability of QD and QDash broad-wavelength gain device is significant for broad wavelength tunability applications in optical communications [5], metrology [6], imaging [7] as well as spectroscopy and sensing [8].

The motivation of this work stems from the potential need to process wavelength information detected from the transmission or reflection of such broad-wavelength, and also due to the need to design new optical component to implement various optical functions in PIC with a small foot-print. The broadwavelength emission is particularly suitable for application in optical sensing, such as in the compact optical coherent tomography (OCT) [7]. Specifically, the transverse magnetic (TM) mode is the preferred evanescent field for such application compared to the transverse electric (TE) field [9]. Hence, in this study, we propose the design of a Cu-based TM- pass polarizer, which has a high pass characteristic suitable for processing the O-, E-, S-, C-, L-, U-band (OESCLU-band) wavelength information, while suppressing the short wavelength light, including the UV, visible and near infra-red regimes.

\section{Structure CONSIDERATIONS, Results AND Discussions}

In this study, a transverse-magnetic-pass (TM-pass) copper $(\mathrm{Cu})$ polarizer is proposed and analyzed using two-dimensional Method-of-Lines beam-propagation model (BPM). The transmission mode TM-pass polarizer has a Si-core/Cufilter/Si-core configuration along the longitudinal direction or direction of wave propagation (see inset in Fig. 1 showing the plan view of the structure). The $300 \mathrm{~nm}$ wide Si-core sections are the input and output waveguides, while the TM-pass $\mathrm{Cu}$ filter (the two orange strips in Fig. 1 inset) consists of two 100 $\mathrm{nm} \times 100 \mathrm{~nm} \mathrm{Cu}$ strips, separated by $100 \mathrm{~nm} \mathrm{SiO}_{2}$ in between. The $\mathrm{Si}$-core/Cu-filter/Si-core structure is protected by $\mathrm{SiO}_{2}$ along the transverse direction as shown in the inset.

The use of double $\mathrm{Cu}$-strip has the following advantages. Firstly, the $\mathrm{Cu}$ strips can confine the long wavelength OESCLU-band using a small physical size structure of up to $300 \mathrm{~nm}$, without which much severe optical leakage is expected. Secondly, it can be potentially implemented as an active device by having two extended electrodes for electrooptical modulation design. As discussed in the above, the structure can hence serve as the basic optical module, of which various optical functions can be implemented.

In the simulation, the $\mathrm{Cu}$ optical constants are taken from reference [10] as shown in Table I. The $\mathrm{Cu}$-filter is launch with the fundamental mode of the input wave, which was swept from 0.4-1.6 $\mu \mathrm{m}$, and the corresponding complex refractive indexes or optical constants $(n-k)$ for $\mathrm{Cu}$ at various wavelengths were used to compute the $\mathrm{TM}_{0}$ and $\mathrm{TE}_{0}$ mode transmissivity. The simulation is performed considering the plan view of the $2 \mathrm{D}$ structure in the Fig. 1. inset, which has a longitudinal discontinuity along the $\mathrm{Si}$-core/Cu-filter/Si-core slabs. The details of the simulation can be found in reference [11].

Fig. 1 shows that the proposed polarizer exhibits a simulated high-pass filter characteristics, with $\mathrm{TM}_{0}$ and $\mathrm{TE}_{0}$ mode transmissivity of $>70 \%$ and $<5 \%$, respectively, in the 
wavelength regime of $1.2-1.6 \mu \mathrm{m}$. The polarization extinction ratio (PER) given by $10 \log _{10}\left(\mathrm{PTM}_{0}\right) /\left(\mathrm{PTE}_{0}\right)$ is $+11.5 \mathrm{~dB}$ across the high-pass wavelength regime. The simulated images for the $\mathrm{TM}_{0}$ and $\mathrm{TE}_{0}$ modes propagation are shown in Fig. 2(a) and Fig. 2(b), respectively, which exhibits the corresponding $\mathrm{TM}_{0}$-pass and $\mathrm{TE}_{0}$-reject characteristics at $1.55 \mu \mathrm{m}$ wavelength. The proposed TM-pass element is practical for SOI photonic integrated circuits because it can be potentially implemented using the existing $\mathrm{Cu}$ Damascene process using the silicon foundry technology. The current simulation does not consider the required $\mathrm{Cu}$ diffusion barrier in practical design, which will be included in future simulation studies and actual implementation.

This TM polarizer configuration is an alternative to the other reported polarizer based on corrugated waveguide [12], photonics crystal waveguide [13], slotted waveguide [14], and sandwiched waveguide [15] structures. The major advantage of our proposed waveguide structure in the $\mathrm{TM}_{0}$ and $\mathrm{TE}_{0}$ modes segregation is evident from the smallest reported foot-print and sub-micron length features. This is beneficial for high density photonic integrated circuits.

Although this study focuses on the passive component implementation, the fundamental building block can also be modified for electro-optical modulation by extending the $\mathrm{Cu}$ metal layers to form two separate electrodes for external biasing of an embedded semiconductor. The feasibility of integrating a small foot-print structure to realize various optical functions, such as a high-wavelength pass filter, a TMpolarizer and potentially an electro-optical modulator with CMOS process compatible features further elucidates the significance of this work.

TABLE I

THE OPTICAL CONSTANTS USED FOR THE SIMULATION WORK IN THIS PAPER.

\begin{tabular}{|c|c|c|}
\hline $\begin{array}{l}\text { Wavelength } \\
(\mathrm{nm})\end{array}$ & $\boldsymbol{n}$ & $\boldsymbol{k}$ \\
\hline 397 & 1.32 & 2.12 \\
\hline 413 & 1.28 & 2.21 \\
\hline 431 & 1.25 & 2.31 \\
\hline 451 & 1.24 & 2.40 \\
\hline 471 & 1.25 & 2.48 \\
\hline 496 & 1.22 & 2.56 \\
\hline 521 & 1.18 & 2.61 \\
\hline 549 & 1.02 & 2.58 \\
\hline 582 & 0.7 & 2.70 \\
\hline 617 & 0.3 & 3.21 \\
\hline 660 & 0.22 & 3.75 \\
\hline 705 & 0.21 & 4.21 \\
\hline 756 & 0.24 & 4.67 \\
\hline 821 & 0.26 & 5.18 \\
\hline 892 & 0.3 & 5.77 \\
\hline 984 & 0.32 & 6.42 \\
\hline 1088 & 0.36 & 7.22 \\
\hline 1216 & 0.48 & 8.25 \\
\hline 1393 & 0.6 & 9.44 \\
\hline 1610 & 0.76 & 11.12 \\
\hline
\end{tabular}

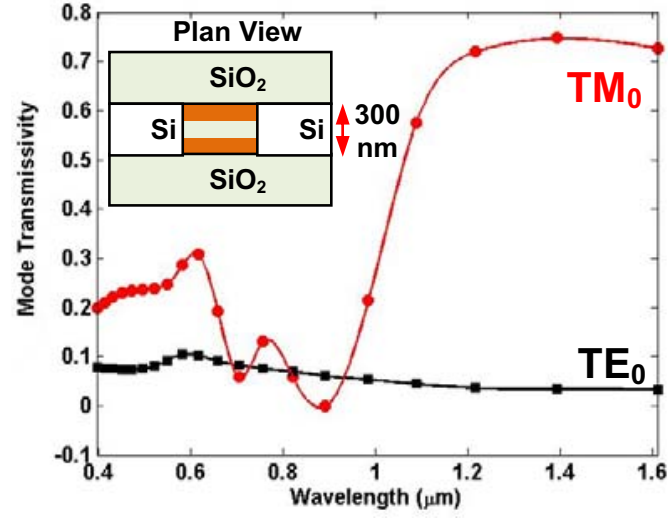

Fig. 1. Mode transmissivity of $\mathrm{TM}_{0}$ and $\mathrm{TE}_{0}$ versus wavelength for the TMpass $\mathrm{Cu}$ polarizer. The inset shows the plan view of the core-Si/Cu-filter/Sicore configuration under study. (a)

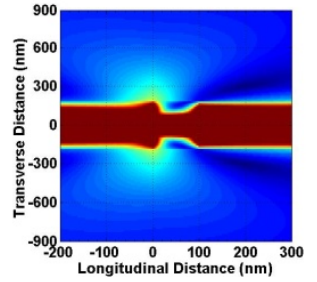

(b)

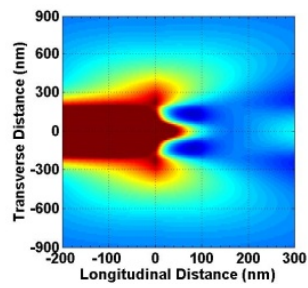

Fig. 2. Simulated images of: (a) the $\mathrm{TM}_{0}$ fundamental mode propagation at $1.55 \mu \mathrm{m}$ wavelength; and (b) simulated image of the $\mathrm{TE}_{0}$ fundamental mode propagation at $1.55 \mu \mathrm{m}$ wavelength. The $100 \mathrm{~nm} \mathrm{Cu}$ strip spans from $0-100$ $\mathrm{nm}$ along the longitudinal direction.

\section{CONCLUSIONS}

We propose here a CMOS compatible high pass TMpolarizer. The simulated results showed the $\mathrm{TM}_{0}$ and $\mathrm{TE}_{0}$ mode transmissivity of $>70 \%$ and $<5 \%$, respectively, in the wavelength regime of $1.2-1.6 \mu \mathrm{m}$. The proposed structure has polarization extinction ratio (PER) of $+11.5 \mathrm{~dB}$ across the highpass wavelength regime. To the best of the authors' knowledge, this is the smallest footprint CMOS-platform compatible TMpolarizer.

\section{ACKNOWLEDGMENT}

The work is supported by a joint program between KAUST and University of Michigan, Ann Arbor, under KAUST Academic Excellence Alliance (AEA) 2010 Grant.

\section{REFERENCES}

[1] S. E. Miller, "Integrated Optics - An Introduction", Bell System Technical Journal, 48, pp.2059, 1969.

[2] D. H. Broaddus, M. A. Foster, I. H. Agha, J. T. Robinson, M. Lipson, and A. L. Gaeta, "Silicon-waveguide-coupled high-Q Chalcogenide Microspheres", Optics Express, 17(8), pp. 5998, 2009.

[3] H. S. Djie, C. L. Tan, B. S. Ooi, J. C. M. Hwang, X. M. Fang, Y. Wu, J. M. Fastenau, W. K. Liu, G. T. Dang, and W. H. Chang, "Ultrabroad Stimulated Emission from Quantum-dash Laser", Appl. Phys. Lett., 91, pp. $111116,2007$.

[4] B. S. Ooi, H. S. Djie, Y. Wang, C. L. Tan, J. C. M Hwang, X. M. Fang, J. 
M. Fastenau, A. K. Liu, G. T. Dang, and W. H Chang, "Quantum dashes on InP Substrate for Broadband Emitter Applications", IEEE J. Sel. Top. Quantum Electron., 14, pp. 1230-1238, 2008.

[5] B. C. Collings, M. L. Mitchell, L. Boivin, and W. H. A. Knox, "1021 Channel WDM System”. Opt. Photon. News, 11, pp. 31-35, 2000.

[6] D. J. Jones, S. A. Diddams, J. K. Ranka, A. Stentz, R. S. Windeler, J. L. Hall, S. T. Cundiff, "Carrier-Envelope Phase Control of Femtosecond Mode-Locked Lasers and Direct Optical Frequency Synthesis", Science, 288(5466), pp. 635 - 639, 2000.

[7] D. Huang, E. A. Swanson, C. P. Lin, J. S. Schuman, W. G. Stinson, W. Chang, M. R. Hee, T. Flotte, K. Gregory, C. A. Puliafito, and al. et., "Optical Coherence Tomography", Science, 254(5035), pp.1178-1181, 1991.

[8] H. I. Schiff, et al., Chapter 5, Wiley Interscience, New York, 1994.

[9] A.Densmore, D.-X. Xu, P. Waldron, S. Janz, P. Cheben, J. Lapointe, A. Delage, B. Lamontagne, J. H. Schmid, and E. Post, "A Silicon-on insulator Photonic Wire Based Evanescent Field Sensor", IEEE J. Quantum Electron., 18(23), pp. 2520-2522, 2006.

[10] P. B. Johnson, and R. W. Christy, "Optical Constants of the Noble Metals", Physical Review B, 6(12), pp. 1972.

[11] H. A. Jamid, M. Z. M. Khan, and M. Ameeruddin, "A Compact 90。 Three-Branch Beam Splitter Based on Resonant Coupling", Journal of Lightwave Technology, 23(11), pp. 3900, 2005.

[12] M. A. Khan, and H. A. Jamid, "Analysis of TM Pass Reflection Mode Optical Polarizer Using Method of Lines", Proceedings of the $10^{\text {th }}$ IEEE International Conference on ICECS, 2, pp. 555-558, 2003.

[13] Y. H. Cui, Q. Wu, E. Schonbrun, M. Tinker, J-B. Lee, and W. Park, "Silicon-based 2D Slab Photonic Crystal TM Polarizer in Telecommunication Wavelength", IEEE Photonics Technology Letters, 20(8), pp. 641-643, 2008.

[14] C. H. Chen, L. Pang, C. H. Tsai, U. Levy, and Y. Fainman, "Compact and Integrated TM-pass Waveguide Polarizer”, Optics Express, 13(14), pp. 5347, 2005.

[15] Q. Wang, and S. T. Ho, "Ultracompact TM-pass Silicon Nanophotonic Waveguide Polarizer and Design”, IEEE Photonics Journal, 2(1), pp. 49, 2010. 